\title{
Rol de los plexos ganglionares en la génesis y el mantenimiento de la fibrilación auricular y la modulación del sistema nervioso autónomo como parte del tratamiento de la fibrilación auricular
}

\section{Role of ganglionic plexuses in the genesis and maintenance of atrial fibrillation and modulation of the autonomic nervous system as part of the atrial fibrillation therapy}

\author{
Ana Lucía Carvajal-Paz \\ IDIME, Los Comuneros Hospital Universitario de Bucaramanga, Bucaramanga, Colombia
}

Recibido el 3 de octubre de 2016; aceptado el 6 de octubre de 2016

Disponible en Internet el 10 de noviembre de 2016

\section{Introducción}

La fibrilación auricular es la arritmia sostenida más común en la práctica clínica y es la causa de un tercio de las hospitalizaciones por arritmias cardiacas. Dichas hospitalizaciones siguen en aumento debido al envejecimiento de la población, la mayor prevalencia de las enfermedades crónicas cardiovasculares y la mayor detección de la arritmia mediante dispositivos de monitorización ambulatoria de toda índole ${ }^{1}$.

Estudios clínicos anteriores han sugerido que un aumento del tono parasimpático está implicado en la génesis de al menos algunas formas de fibrilación auricular paroxística ${ }^{2,3}$. Se ha visto que en modelos caninos, la estimulación vagal acorta el período refractario de la fibrilación auricular, aumenta su dispersión y disminuye la longitud de onda de los circuitos reentrantes que facilitan el inicio y mantenimiento de la fibrilación auricular ${ }^{3,4}$.

Correo electrónico: acarva@yahoo.com

\section{Discusión}

Con la adopción de técnicas de ablación con catéter para el tratamiento de las taquiarritmias se ha incrementado el interés en el aprendizaje de la anatomía cardiaca y los electrofisiólogos están prestando más atención a estudiar la morfología y las características arquitectónicas del corazón. Como resultado de este interés ha surgido una nueva ola de investigación para estudiar temas de anatomía cardiaca que no fueron abordados por completo en el pasado. Recientes investigaciones han desentrañado características anatómicas, aspectos arquitectónicos y detalles histológicos de ciertos componentes del corazón, de interés para la comprensión de los sustratos de las taquicardias y su ablación ${ }^{5}$.

Las áreas de las uniones veno-auriculares y adyacentes a las venas pulmonares están ricamente inervadas por los nervios ganglionares procedentes del plexo neural cardiaco. Los subplexos epicárdicos ubicados en las almohadillas de grasa auriculares envían abundantes extensiones.

Las bases electrofisiológicas de la fibrilación auricular requieren de un desencadenante que inicie las arritmias y un 
sustrato que las mantengan. Lo más común es que este detonante sea un latido ectópico que se inicie en los músculos aledaños de las venas pulmonares. Esos detonantes provocan una actividad intrínseca cardiaca en los plexos ganglionares, que a la larga se quedan agrupados en el cruce de la unión veno-auricular izquierda. Estos detonantes y el crecimiento auricular provocan fibrosis e inflamación y sirven con sustratos para mantener las ondas en fibrilación auricular. La persistencia de la fibrilación auricular predispone al desarrollo de otros detonantes y ondas. El proceso se traduce en una perpetuación de la fibrilación auricular. El mantenimiento de un ritmo sinusal puede revertir esos cambios y mecanismos de perpetuación, así como a su vez, el ritmo de fibrilación auricular hace que el paciente tenga más ritmo de fibrilación auricular.

El corazón está influenciado por el control autónomo de los sistemas simpático y parasimpático (vagal), que ejercen su acción a través de los plexos cardíacos que se encuentran en la base del corazón, divididos en dos porciones; una superficial (ventral) y otra profunda (dorsal).

La inervación del corazón está dada por fibras nerviosas autónomas procedentes de los nervios vagos y de los troncos simpáticos ${ }^{6}$.
- Plexo nervioso cardiaco superficial: proveniente del ganglio cervical superior izquierdo (simpático) y del nervio vago izquierdo (parasimpático), formará el plexo pulmonar izquierdo y coronario derecho.

- Plexo nervioso cardiaco profundo: proveniente de los ganglios simpáticos cervicales medio e inferior izquierdos, ganglios simpáticos cervicales superior, medio e inferior derecho, ganglios simpáticos torácicos (comúnmente T1T4 y en algunos casos T5 y T6) de ambos lados y del nervio vago derecho, formará el plexo pulmonar derecho, coronario izquierdo y parte del coronario derecho.

- Ramas comunicantes: entre ambos plexos cardiacos.

\section{Inervación del pericardio}

- El pericardio seroso (formado por una sola capa de células mesoteliales) se inerva por ramas de los plexos subsidiarios del plexo cardíaco, que tienen fibras simpáticas, parasimpáticas y aferentes. El pericardio parietal se inerva por fibras sensitivas que viajan en su mayoría por los nervios frénicos, aunque también por los nervios intercostales. También recibe fibras simpáticas y parasimpáticas del plexo cardíaco.
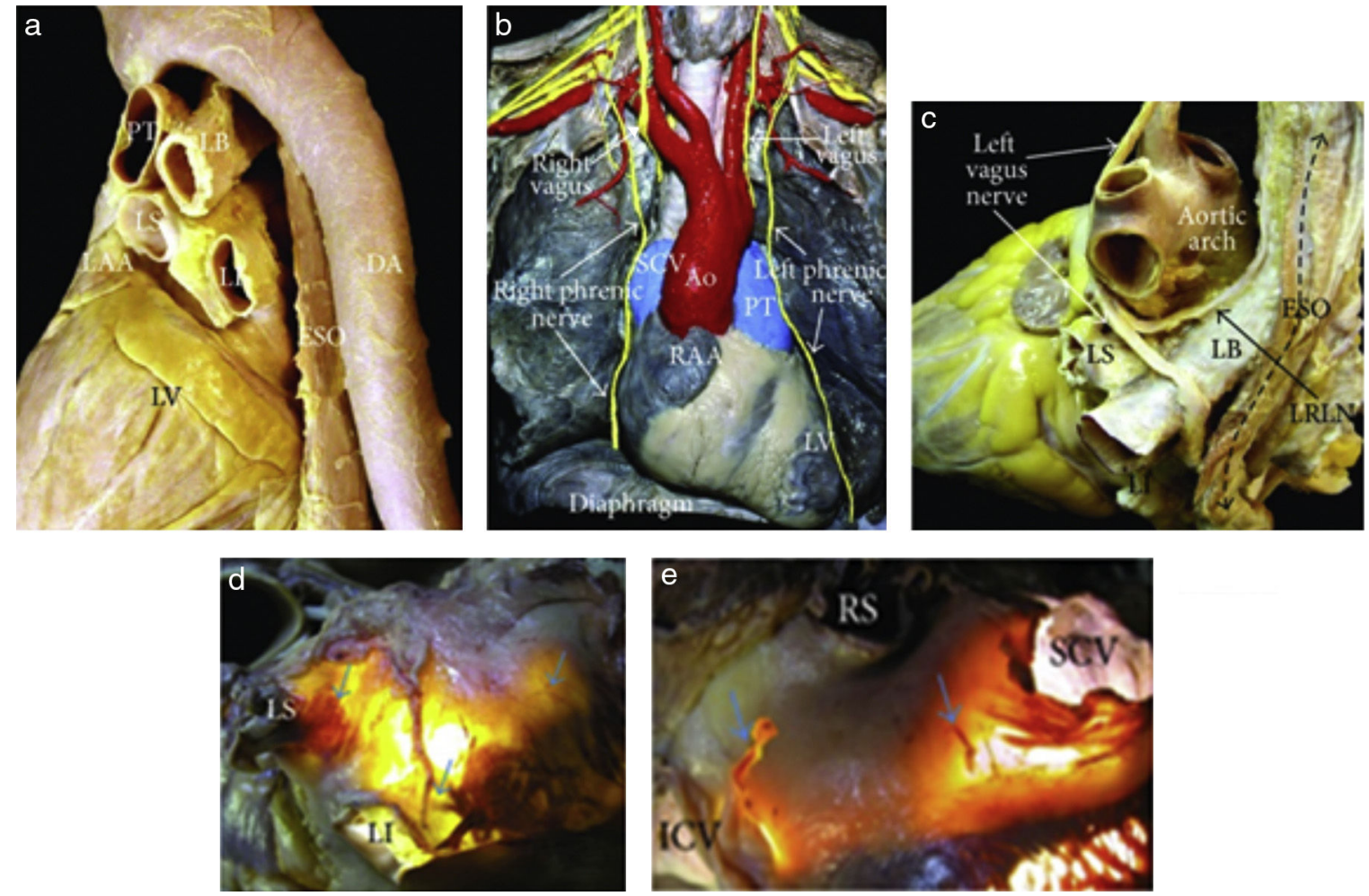

Figura 1 (a) Vista en perspectiva posterolateral izquierda que muestra el curso del esófago y descendente de la aorta respecto a la aurícula izquierda. (b) En disección de un cadáver se ve desde la parte frontal, muestra el curso de los nervios frénicos derechos e izquierdos y los nervios vagos. (c) Disección del nervio vago izquierdo y su rama del laríngeo recurrente izquierdo, que desciende sobre el techo de la aurícula izquierda. (d, e). Transiluminación del techo de la aurícula izquierda y el surco interauricular posterior en donde se aprecian los plexos ganglionares epicárdicos de la acetilcolinesterasa de colores (flechas azules) que se extienden a la superficie de la unión venoauricular izquierda en (d) y región intercaval en $(e)^{5}$. Ao = aorta, $L B=$ bronquio izquierdo, DA $=$ aorta descendente, $\mathrm{ESO}$ = esófago, ICV = vena cava inferior, $\mathrm{LI}$ = vena pulmonar inferior izquierda, $\mathrm{LRLN}=$ nervio laríngeo recurrente izquierdo, $\mathrm{LS}=$ vena pulmonar superior izquierda, $\mathrm{LV}=$ ventrículo izquierdo, $\mathrm{PT}=$ tronco pulmonar, $\mathrm{RAA}=$ apéndice auricular derecho, $\mathrm{RI}=\mathrm{vena}$ pulmonar inferior derecha, RS = vena pulmonar superior derecha, y SCV = vena cava superior. 
- Inervación autónoma del corazón: la inervación parasimpática del corazón está dada por neuronas preganglionares que se ubican en el núcleo dorsal del vago. Las fibras preganglionares de estas neuronas constituyen el nervio vago que se dirige al corazón para hacer sinapsis en las neuronas postganglionares del plexo cardíaco ubicado en el corazón. Las fibras postganglionares tienen su sinapsis en los nodos sinusal, auriculoventricular y en las arterias coronarias. La activación del parasimpático produce disminución de la frecuencia cardíaca (bradicardia), así como de la fuerza de contracción del corazón, y constricción de las arterias coronarias. La inervación simpática está dada por neuronas preganglionares del asta lateral de los segmentos torácicos T2-T4. Las fibras de estas neuronas abandonan la médula por los nervios raquídeos correspondientes, ascienden por la cadena simpática latero-vertebral para hacer su sinapsis en los ganglios cervicales superior, medio e inferior y en los dos a tres primeros ganglios torácicos. Desde allí, las fibras postganglionares transcurren por las ramas cardíacas superior, media e inferior de la porción cervical del tronco simpático y algunas ramas cardíacas torácicas. Todas ellas llegan al plexo cardíaco para inervar los nódulos sinusal, auriculoventricular y las arterias coronarias. La activación del simpático produce aumento de la frecuencia cardíaca (taquicardia), aumento de la fuerza de contracción del músculo cardíaco y dilatación de las arterias coronarias ${ }^{5}$. El acceso pericárdico a la aurícula izquierda y a las venas pulmonares está limitado por el hecho de que el saco pericárdico se dobla alrededor del corazón. El acceso a la parte anterior de la aurícula izquierda se puede hacer a través del seno transverso, que se extiende entre la parte posterior de la aorta ascendente y la bifurcación del tronco pulmonar y la parte delantera de las cámaras auriculares $^{5}$ (fig. 1).

De acuerdo con los criterios establecidos para distinguir los plexos ganglionares, son extensiones epicárdicas de los nervios del mediastino que entran en el corazón a través de sitios discretos en el hilio del corazón y proceden por separado en las regiones de inervación por siete caminos. En general, la aurícula derecha humana es inervada por dos subplexos, la aurícula izquierda por tres, el ventrículo derecho por uno y el ventrículo izquierdo por tres. La mayor densidad de ganglios epicárdicos está ubicada cerca del hilio del corazón, especialmente en las superficies dorsales y dorsolaterales de la aurícula izquierda, donde se encuentra hasta el $50 \%$ de todos los ganglios cardiacos ${ }^{7}$ (fig. 2).

Las áreas de las uniones veno-auricular y adyacentes a las venas pulmonares son también muy inervadas por los nervios ganglionares procedentes del plexo neural cardiaco. Los subplexos epicárdicos ubicados en las almohadillas de grasa auriculares envían extensiones nerviosas abundantes ${ }^{6,9}$.

\section{Subplexos ganglionares epicárdicos}

El sistema nervioso autónomo del corazón también parece desempeñar un papel de cierta relevancia en la patogénesis de la fibrilación auricular paroxística (fig. 3). Se ha

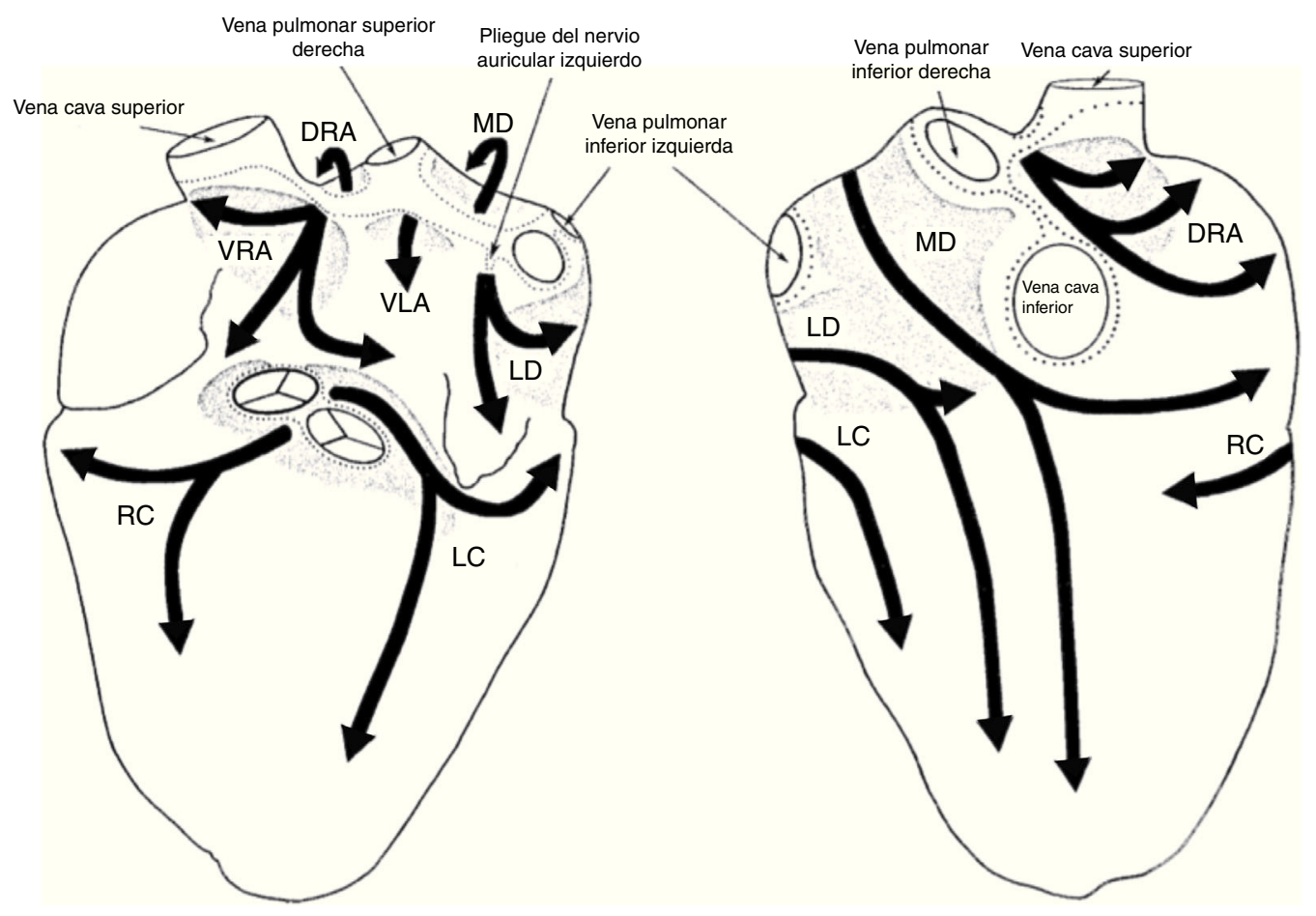

Figura 2 Esquema que resume la descripción de la disposición y las regiones de inervación de los siete subplexos epicárdicos en humanos. Izquierda: Vista ventral. Derecha: Vista dorsal. Los subplexos ganglionares se representan mediante sombras. Las líneas de puntos indican los límites del hilio corazón. VRA: subplexos ganglionares de la aurícula derecha; DRA: subplexos dorsales de la aurícula derecha; MD: subplexo ganglionar dorsal medio; VLA: subplexo ganglionar de la aurícula izquierda; LD: subplexo ganglionar dorsal izquierdo; RC: subplexo ganglionar coronario derecho; LC = subplexo ganglionar coronario izquierdo ${ }^{7}$. 


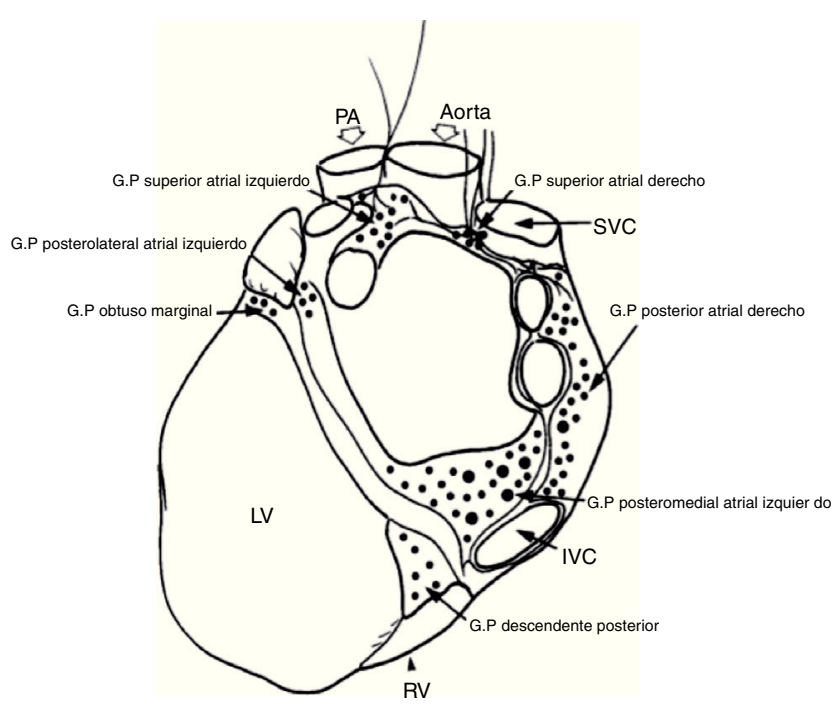

Figura 3 Localización de los plexos ganglionares ${ }^{8}$. GP: plexo ganglionar, PA: arteria pulmonar, SVC: vena cava superior, IVC: vena cava inferior, RV: ventrículo derecho, LV: ventrículo izquierdo. observado que, con frecuencia, los episodios de fibrilación auricular paroxística van precedidos de un aumento del tono simpático y parasimpático. Existen además evidencias experimentales de que la estimulación del sistema nervioso autónomo cardíaco, especialmente el colinérgico, favorece la actividad eléctrica ectópica y los fenómenos de reentrada en la región de las venas pulmonares ${ }^{9}$. Por tanto, la técnica de ablación para el tratamiento de la fibrilación auricular paroxística deberá incluir ineludiblemente, al menos, la creación de cicatrices transmurales en la aurícula izquierda que engloben los orificios de drenaje de las venas pulmonares, y, quizás, según el tamaño de la aurícula izquierda la pared posterior de la misma. Los objetivos buscados con este procedimiento serían, por una parte, lograr un aislamiento completo de los focos iniciadores de la arritmia del resto del miocardio auricular y, por otra, la destrucción de la inervación simpática y parasimpática local procedente de estos vasos. Según sea fibrilación auricular paroxística aislada o paroxística asociada a cardiopatía estructural, la efectividad de los diferentes patrones varía significativamente ${ }^{9,10}$. La influencia del sistema nervioso autónomo (SNA) sobre la activación y la perpetuación de la fibrilación auricular está bien establecida. Las variaciones del tono autonómico se han asociado con paroxismos de fibrilación auricular y tanto la activación simpática como la parasimpática pueden ser proarrítmicas acortando el
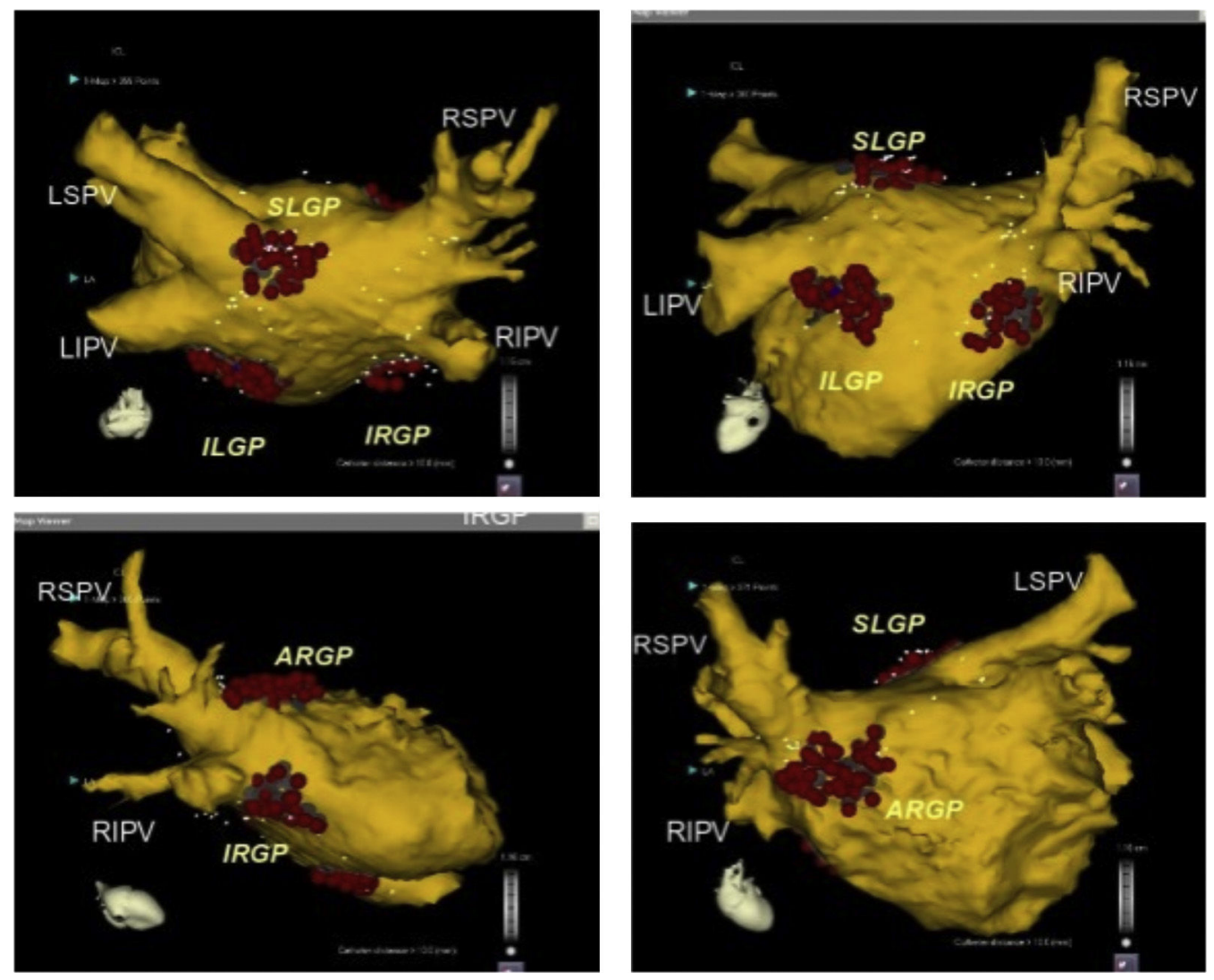

Figura 4 Posición anatómica de los plexos ganglionares más importantes objetivos de la ablación con catéter. Se presume que los plexos ganglionares son seccionados $1-2 \mathrm{~cm}$ fuera de las uniones de la unión AI-VP en los siguientes sitios: zona superolateral de la aurícula izquierda (superior izquierda SLGP GP), área superoanterior derecha (anterior derecho GP-ARGP), a la izquierda zona inferoposterior (inferior izquierda GP-ILGP) y a la derecha zona inferoposterior (inferior derecha GP-IRGP). Otro GP (quid GP) la zona inferoposterior entre la ILGP y IRGP ${ }^{16}$. 


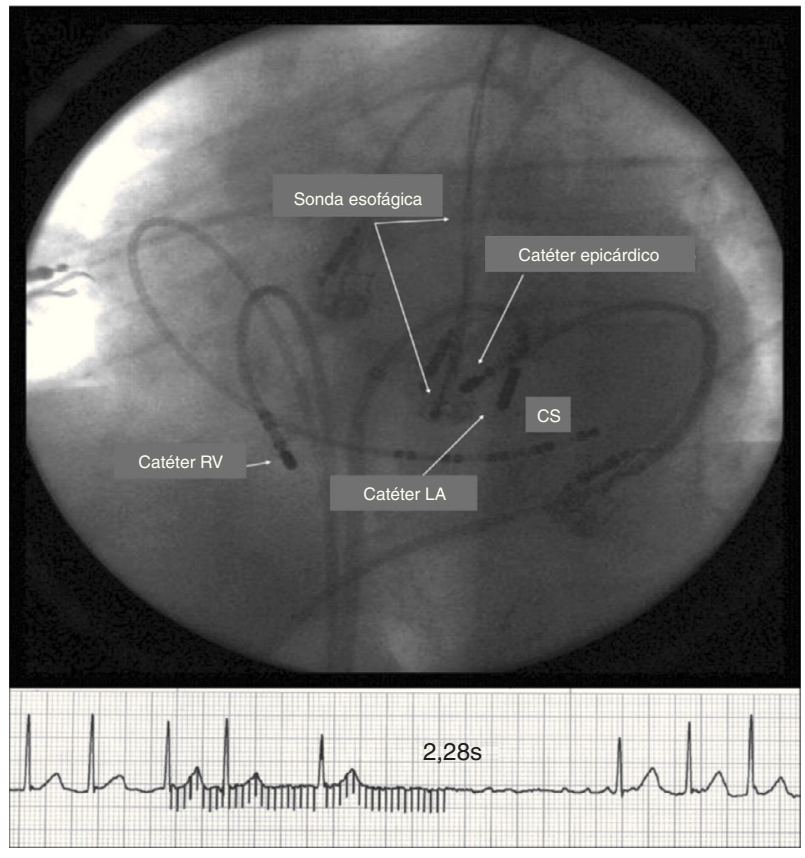

Figura 5 Respuesta vagal (pausa, mayor de 2,28 segundos) durante estimulación epicárdica de $\mathrm{HF}(20 \mathrm{~Hz})$. Nótese la estrecha relación entre la sonda esofágica y el catéter epicárdico (punta de $4 \mathrm{~mm}$ ). Para evitar el daño del esófago, la radiofrecuencia se aplica desde el catéter endocárdico (punta de $8 \mathrm{~mm}$ ). El catéter epicárdico fue ubicado entre el catéter endocárdico y la sonda esofágica de temperatura. La temperatura esofágica más alta registrada fue de $37,5^{\circ} \mathrm{C}$, y el reflejo vagal por estimulación epicárdica fue suprimido totalmente. CS: seno coronario; LA catheter: catéter endocárdico auricular y RV catheter, catéter en el ventrículo derecho ${ }^{24}$.

período refractario auricular. Los estudios iniciales sobre la denervación vagal parcial a través del epicardio o la ablación de almohadilla de grasa, indicaron que pueden prevenir la fibrilación auricular ${ }^{11,12}$. El ligamento de Marshall es un haz pericárdico de la aurícula izquierda neuromuscular, rico en la inervación simpática, que se ha asociado con la génesis de taquiarritmias atriales y fibrilación auricular ${ }^{10,13}$. La ablación epicárdica del ligamento de Marshall en humanos (a través del seno coronario) ${ }^{14}$ o la ablación endocárdica en el sitio de inserción del haz de Marshall puede terminar la fibrilación auricular. Se sabe que la denervación simpática o parasimpática selectiva puede no ser factible. Ambos elementos, simpático y parasimpático, residen en los cuatro plexos ganglionares principales de la aurícula izquierda ${ }^{14}$; las lesiones de ablación pueden afectar inevitablemente ambos componentes del sistema nervioso autónomo (fig. 4).

La ablación de los plexos ganglionares (plexos ganglionares) logra la denervación autonómica al afectar tanto a los componentes simpático y parasimpático del SNA. La ablación de los plexos ganglionares parece ser segura, y mejora los resultados de aislamiento de las venas pulmonares en pacientes con fibrilación auricular persistente y paroxística. Dicha ablación puede llevarse a cabo de manera endocárdica o epicárdica, es decir, durante el procedimiento de Maze (laberinto) o enfoque toracoscópico ${ }^{15}$ (fig. 5). Múltiples estudios sugieren que los plexos ganglionares pueden ser uno de los sustratos implicados en la perpetuación de los circuitos reentrantes de la fibrilación auricular, y por tanto la eliminación de estos estímulos autonómicos puede conducir a mayor ausencia de arritmias.

Los plexos ganglionares se identifican en el laboratorio de electrofisiología mediante estimulación a alta frecuencia (HFS) y la inducción de respuestas vagales en las aurículas $^{16,17}$. Se estimula a $1.200 \mathrm{lpm}(20 \mathrm{~Hz})$ con ancho de pulso de $10 \mathrm{~ms}$ a $12-24 \mathrm{v}$. Una respuesta vagal eferente predominante se define como inducción de bloqueo $\mathrm{AV}(>2 \mathrm{~s})$ e hipotensión o prolongación del intervalo $\mathrm{R}-\mathrm{R}>50 \%$ durante fibrilación auricular, después de un segundo de aplicación de la estimulación de alta frecuencia. Sin embargo, el método generalmente conlleva molestia por la anestesia general, puesto que pacientes conscientes no pueden tolerar más de $15 \mathrm{v}$. Además, se ha demostrado recientemente que la ablación anatómica, es decir dirigida a las áreas de los plexos ganglionares en la aurícula izquierda (fig. 6), tiene mejores resultados clínicos en pacientes con fibrilación auricular paroxística $^{15}$.

La ablación aislada de los plexos ganglionares se ha empleado con éxito variable para la fibrilación auricular paroxística y persistente. En la fibrilación auricular paroxística, la supervivencia libre de arritmia durante el primer año después del procedimiento osciló entre 26 y $77 \%{ }^{18,19}$; se han reportado tasas de éxito de < $40 \%$ para la fibrilación auricular persistente después de un procedimiento único. La ablación de los plexos ganglionares en combinación con el aislamiento de las venas pulmonares, produjo mejores resultados de aislamiento con tasas de éxito de hasta un $80 \%{ }^{18-20}$.

Estudios demuestran que en poblaciones con fibrilación auricular paroxística de origen vagal, la ablación anatómica de los plexos ganglionares en la aurícula derecha es eficaz en alrededor del $70 \%$ de los pacientes ${ }^{21,22}$.

\section{Denervación autonómica}

Se realiza ablación aplicando radiofrecuencia (la energía de radiofrecuencia a $60^{\circ}, 30-70 \mathrm{~W}$ durante 30 - 60 segundos) en los sitios anatómicos de los plexos ganglionares de la aurícula derecha (figs. 6A y B), la zona superoposterior (superior plexos ganglionares $A D$ junto a la unión de la vena cava superior [SVC] y la superficie posterior de la $A D)$, la zona media posterior (posterior $A D \mathrm{GP}$, superficie posterior de la $A D$ junto el surco interauricular), la zona inferoposterior (plexo ganglionar colocado entre la vena cava inferior, el ostium del seno coronario y cerca del surco auriculoventricular). La ablación se realiza hasta que la actividad eléctrica se reduzca significativamente (electrograma bipolar de pico a pico $<0,05 \mathrm{mV}$ ) y el reflejo vagal desaparezca. Se ha visto que los CFAE (complex fractionated atrial electrograms) se encuentran principalmente en la pared posterior y septal en la proximidad de sitios de los plexos ganglionares. Durante la ablación de los plexos ganglionares se reducen significativamente los CFAE (figs. 7 y 8 ).

Se ha demostrado que la estimulación de los plexos ganglionares, asociada con una respuesta parasimpática causada por la liberación de acetilcolina, aumenta la vulnerabilidad a fibrilación auricular por acortamiento del período refractario de las venas pulmonares y aumento de la 
A

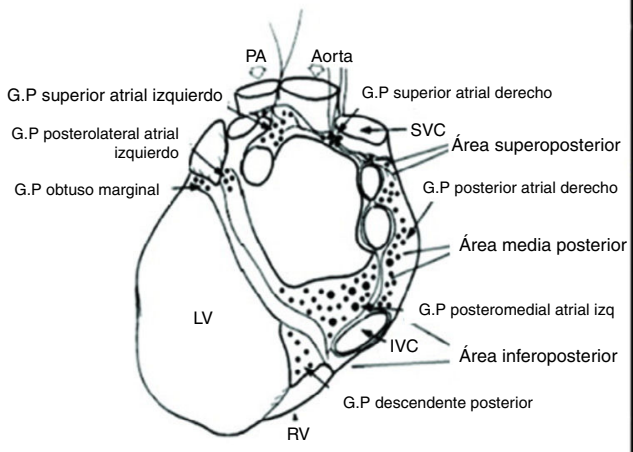

$\mathrm{B}$

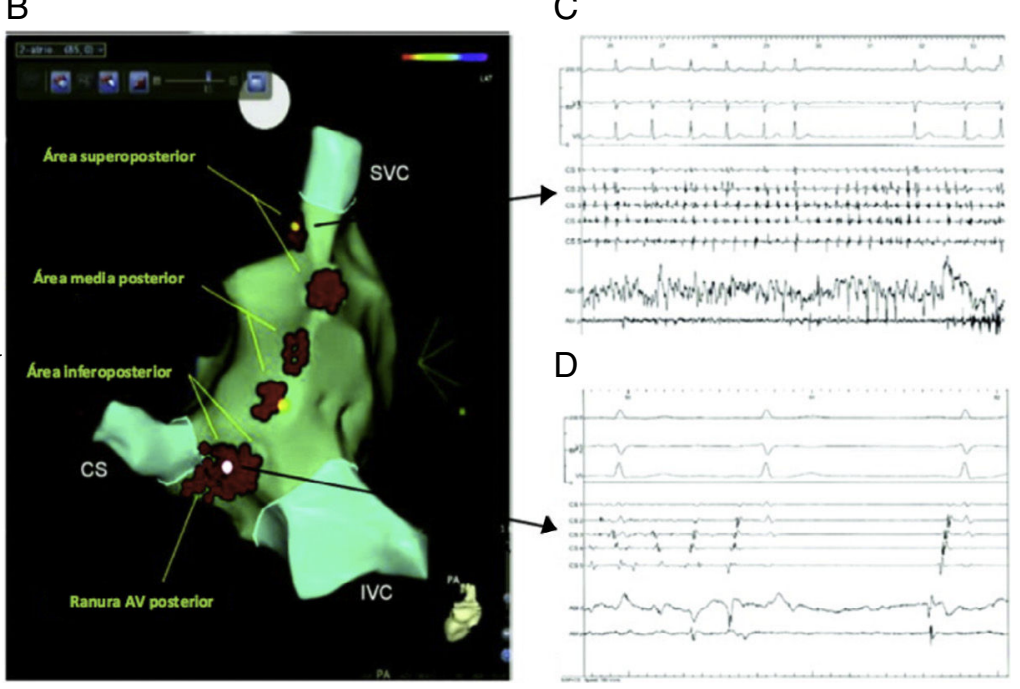

Figura 6 A, Vista posterior del corazón humano, en la que se ilustra la ubicación de los principales plexos ganglionares auriculares (PG). B, CARTO 3 mapa anatómico de la aurícula derecha (RA) (proyección posteroanterior) en un caso de ablación anatómica de plexos ganglionares. Los puntos rojos representan sitios de pulso de radiofrecuencia; los amarillos, sitios de respuesta parasimpática provocada durante la aplicación de radiofrecuencia; y los blancos, sitios donde la fibrilación auricular (FA) terminó durante la ablación. CS: seno coronario; IVC: vena cava inferior; SVC: vena cava superior; y AV: auriculoventricular. C, respuesta parasimpática evocada durante la ablación anatómica. D, restauración del ritmo sinusal durante la ablación anatómica (I, V1 y V6, ECG de superficie; CS1-CS5, seno coronario distal y proximal; $A B L d / p$, bipolar distal y proximal del catéter de ablación; y BP: presión arterial).
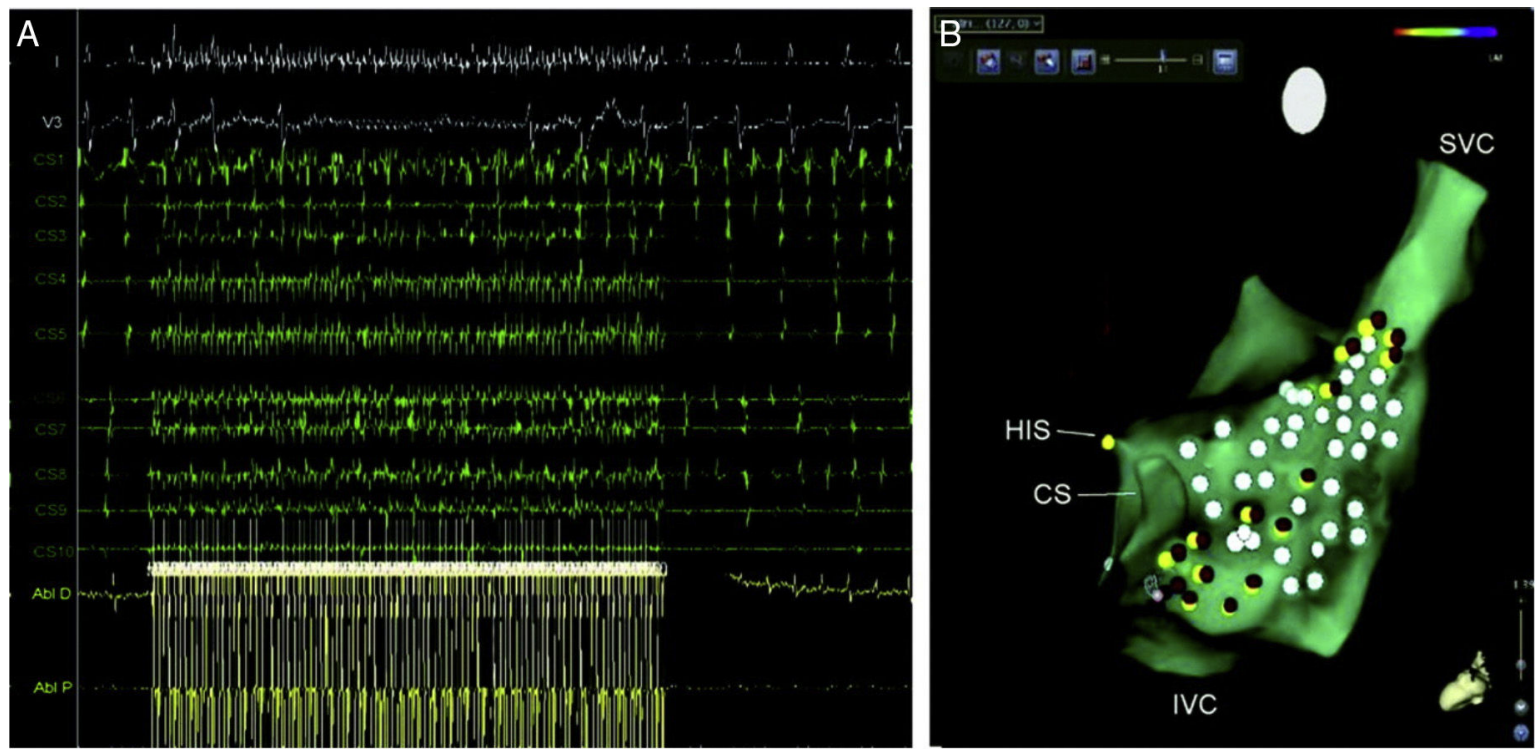

Figura 7 A. Respuesta parasimpática evocada durante la estimulación de alta frecuencia (HFS) cerca del ostium del seno coronario (y V3, superficie ECG; CS1-CS10, seno coronario distal y proximal; ABL d/p, bipolar cartografía distal y proximal y catéter de ablación). B. CARTO 3 mapa anatómico de aurícula derecha (RA) (proyección lateral) en un caso de ablación selectiva del plexo ganglionar (GP). Puntos rojos representan sitios de pulso de radiofrecuencia cerca de lugares de HFS-positiva (puntos amarillos). Puntos blancos representan sitios de HFS-negativo. SU indica proximal del haz de His; CS, del seno coronario; Vena cava inferior, vena cava inferior; SVC, vena cava superior; y AV, auriculoventricular ${ }^{22}$.

dispersión de la refractariedad ${ }^{23}$. Estudios han comprobado que la sola ablación anatómica de los ganglios linfáticos en la aurícula derecha ha demostrado ser efectiva en aproximadamente un $70 \%$ de los pacientes sin realizar la desconexión de las venas pulmonares. Estos resultados confirman que la denervación vagal puede abolir la fibrilación auricular, según lo sugieren datos experimentales y clínicos. Conviene destacar que estos hallazgos se han proyectado en un subgrupo específico de pacientes sin signos de cardiopatía estructural y con episodios de fibrilación auricular de predominio del tono vagal, como ocurre durante el sueño, después de las comidas, tos entre otros. Estas características identifican a 

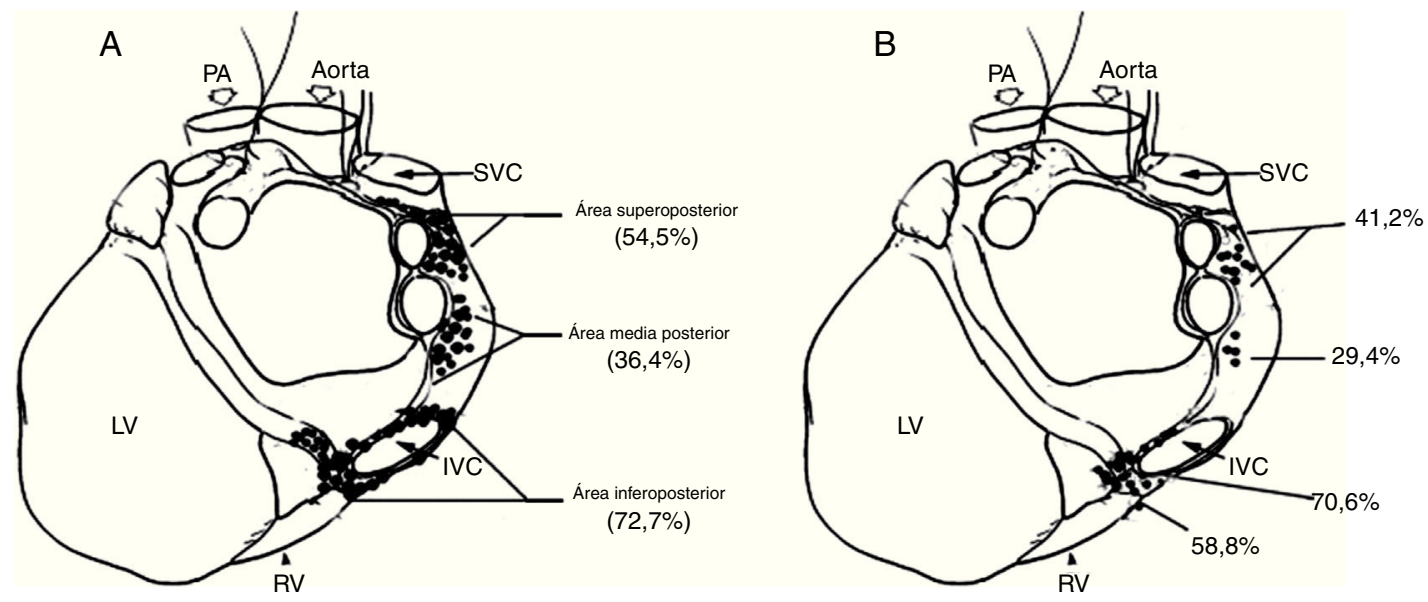

Figura 8 A, distribución de sitios donde la aplicación de radiofrecuencia evocaba una respuesta parasimpática durante la ablación anatómica. B, distribución de sitios donde la estimulación de alta frecuencia evocaba una respuesta parasimpática durante el enfoque selectivo. PA, arteria pulmonar; IVC: vena cava inferior; SVC: vena cava superior; RV: ventrículo derecho; y LV: ventrículo izquierdo ${ }^{22}$.

los pacientes en quienes se debe realizar ablación de los plexos ganglionares de la aurícula derecha.

Enfoques híbridos que combinan por vía toracoscópica el aislamiento de las venas pulmonares con la ablación selectiva de los plexos ganglionares, con o sin ablación del ligamento de Marshall o la amputación de la orejuela de la aurícula izquierda, reportan tasas de éxito a más de un año seguimiento desde 65 a $86 \%$. las estrategias de ablación endocárdica que se dirigen a esas estructuras epicárdicas corren el riesgo de sufrir daños colaterales, mientras que la ablación epicárdica, por ejemplo, mediante técnicas mínimamente invasivas, al menos permite un enfoque más específico (fig. 5).

\section{Conclusión}

Una mejor comprensión de la localización de los plexos ganglionares y el papel de cada uno de ellos en el inicio y mantenimiento de la fibrilación auricular guiará el futuro desarrollo de estrategias de "neuroablación" para los pacientes con fibrilación auricular. Otros estudios en poblaciones más grandes deberán aclarar si la ablación biauricular de los plexos ganglionares solos o en asociación con la desconexión de las venas pulmonares puede aumentar de manera significativa el éxito en la ablación de la fibrilación auricular.

\section{Conflicto de intereses}

Los autores declaran no tener conflicto de intereses.

\section{Bibliografía}

1. Fuster V, Rydén LE, Cannom DS, et al. 2011 ACCF/AHA/HRS focused updates incorporated into the ACC/AHA/ESC 2006 guidelines for the management of patients with atrial fibrillation: a report of the American College of Cardiology Foundation/American Heart Association Task Force on practice guidelines. Circulation. 2011;123:e269-367.
2. Brignole M. Vasovagal syncope and vasovagal disease. Hellenic J Cardiol. 2008;49:61-4.

3. Chen YJ, Chen SA, Tai CT, et al. Role of atrial electrophysiology and autonomic nervous system in patients with supraventricular tachycardia and paroxysmal atrial fibrillation. J Am Coll Cardiol. 1998;32:732-8.

4. Zipes DP, Mihalick MJ, Robbins GT. Effects of selective vagal and stellate ganglion stimulation of atrial refractoriness. Cardiovasc Res. 1974;8:647-55.

5. Sánchez-Quintana D, Doblado-Calatrava M, Cabrera JA, et al. Anatomical Basis for the cardiac interventional electrophysiologist. Biome Rest Int. 2015;547364:24.

6. Richardson R. The making of Mr. Gray's anatomy. Oxford New York: Oxford University Press; 2008.

7. Pauza DH, Skripka V, Pauziene N, et al. Morphology, distribution, and variability of the epicardiac neural ganglionated subplexuses in the human heart. Anatomical Record. 2000;259: 353-82.

8. Calo L, Rebecchi M, Sciarra L, et al. Catheter ablation of right atrial ganglionated plexi in patients with vagal paroxysmal atrial fibrillation. Circ Arrhythm Electrophysiol. 2012;5: 22-31.

9. Sharifov OF, Fedorov VV, Beloshapko GG, et al. Role of adrenergic and cholinergic stimulation in spontaneous atrial fibrillation. JACC. 2004;43:483-90.

10. Bettoni M, Zimmermann M. Autonomic tone variations before the onset of paroxysmal atrial fibrillation. Circulation. 2002;105:2753-9.

11. Olgin JE, Sih HJ, Hanish S, et al. Heterogenous atrial denervation creates substrate for sustained atrial fibrillation. Circulation. 1998;98:2608-14.

12. Coumel P. Paroxysmal atrial fibrillation: a disorder of autonomic tone? Eur Heart J. 1994;15:9-16.

13. Coumel P. Autonomic influences in atrial arrhythmias. J Cardiovasc Electrophysiol. 1996;58:999-1007.

14. Salo MA, Huikuri HV, Seppänen T. Ectopic beats in heart rate variability analysis: effects of editing on time and frequency domain measures. Ann Noninvasive Electrocardiol. 2001;6:5-17.

15. Katritsis DG. Autonomic Denervation for the Treatment of Atrial Fibrillation. Indian Pacing Electrophysiol J. 2011;11:161-6.

16. Lemery R, Birnie D, Tang AS, et al. Feasibility study of endocardial mapping of ganglionated plexuses during catheter ablation of atrial fibrillation. Heart Rhythm. 2006;3:387-96. 
17. Mehall JR, Kohut RM Jr, Schneeberger EW, et al. Intraoperative epicardial electrophysiologic mapping and isolation of autonomic ganglionic plexi. Ann Thorac Surg. 2007;83:538-41.

18. Pokushalov E, Romanov A, Shugayev P, et al. Selective ganglionated plexi ablation for paroxysmal atrial fibrillation. Heart Rhythm. 2009;6:1257-64.

19. Mikhaylov E, Kanidieva A, Sviridova N, et al. Outcome of anatomic ganglionated plexi ablation to treat paroxysmal atrial fibrillation: a 3-year follow-up study. Europace. 2011;13:362-70.

20. Po SS, Nakagawa H, Jackman WM. Localization of left atrial ganglionated plexi in patients with atrial fibrillation. J Cardiovasc Electrophysiol. 2009;20:1186-9.
21. McClelland JH, Duke D, Reddy R. Preliminary results of a limited thoracotomy: new approach to treat atrial fibrillation. J Cardiovasc Electrophysiol. 2007;18:1289-95.

22. Calò L, Rebecchi M, Sciarra L, et al. Catheter ablation of right atrial ganglionated plexi in patients with vagal paroxysmal atrial fibrillation. Circ Arrhythm Electrophysiol. 2012;5:22-31.

23. Hou Y, Scherlag BJ, Lin J, et al. Ganglionated plexi modulate extrinsic cardiac autonomic nerve input: effects on sinus rate, atrioventricular conduction, refractoriness, and inducibility of atrial fibrillation. J Am Coll Cardiol. 2007;50:61-8.

24. Scanavacca M, Pisani C, Hachul D, et al. Selective atrial vagal denervation guided by evoked vagal reflex to treat patients with paroxysmal atrial fibrillation. Circulation. 2006;114:876-85. 